\title{
Congenital adrenal hyperplasia: management during critical illness
}

E Charmandari, E J Lichtarowicz-Krynska, P C Hindmarsh, A Johnston, A Aynsley-Green, C G D Brook

\begin{abstract}
Background-Little is known of the optimal dose and administration schedule of hydrocortisone in critically ill patients with congenital adrenal hyperplasia (CAH) caused by 21-hydroxylase deficiency.

Aim-To determine plasma cortisol concentrations after intravenous administration of hydrocortisone in children with CAH and to relate these to plasma cortisol concentrations achieved by endogenous secretion in the stress of critical illness in previously healthy children.

Methods-Plasma cortisol concentrations were measured in 20 patients with classical CAH (median age 11.2 years, range 6.1-16.4) following intravenous administration of hydrocortisone $15 \mathrm{mg} / \mathrm{m}^{2}$; and in 60 critically ill mechanically ventilated children (median age 2.5 years, range $0.25-16.3$ ) on admission to the paediatric intensive care unit and for 24 hours thereafter.

Results-In the CAH patients, plasma cortisol reached a mean peak of 1648.3 nmol/1 (SD 511.9) within 10 minutes of the intravenous bolus, and fell rapidly thereafter; levels remained greater than 450 nmol/1 for 2.5 hours only. In critically ill children, mean plasma cortisol on admission to the intensive care unit was 727 nmol/1 (SD 426.1). Cortisol concentrations remained raised during the first 24 hours. Conclusions-Critically ill patients with classical CAH may be best managed with a single intravenous hydrocortisone bolus followed by a constant rate infusion of hydrocortisone.

(Arch Dis Child 2001;85:26-28)
\end{abstract}

Keywords: congenital adrenal hyperplasia; critical illness; hydrocortisone; cortisol clearance

Pharmacology, St

Bartholomew's and the

Royal London School

of Medicine and

Dentistry, London

EC1M 6BQ, UK

A Johnston

Correspondence to: Dr E Charmandari, Pediatric and Reproductive

Endocrinology Branch

National Institute of Child

Health and Human

Development, National

Institutes of Health,

Bethesda, Maryland

20892-1583, USA

charmane@mail.nih.gov

Accepted 19 February 2001 administration of a hydrocortisone bolus in a dose of $15 \mathrm{mg} / \mathrm{m}^{2}$ body surface area in CYP21 deficient patients, and to relate these to cortisol concentrations achieved by endogenous secretion in the stress of critical illness in previously healthy children.

Patients and methods

CYP21 DEFICIENT PATIENTS

Twenty children (six boys, 14 girls; median age 11.2 years, range $6.1-16.4$ ) with salt wasting congenital adrenal hyperplasia attending the London Centre for Paediatric Endocrinology were studied prospectively. All patients received standard doses of replacement therapy and displayed adequate clinical and biochemical control. On the day of the study, patients were given their usual dose of mineralocorticoid substitution at 0800; one hour later intravenous hydrocortisone sodium succinate was administered as a bolus in a dose of $15 \mathrm{mg} / \mathrm{m}^{2}$ body surface area. Blood samples for cortisol concentration determination were collected through a second cannula at 10 minute intervals for six hours following the injection of hydrocortisone. Blood samples were centrifuged, separated, and stored at $-20^{\circ} \mathrm{C}$ prior to assay.

CRITICALLY ILL PATIENTS

Sixty critically ill mechanically ventilated but previously healthy children (27 boys, 33 girls; median age 2.5 years, range $0.25-16.3$ ) were recruited from the paediatric intensive care unit at Great Ormond Street Hospital and St Mary's Hospital, London. The primary diagnoses necessitating admission to the intensive care unit included meningococcal septicaemia $(n=29)$, lower respiratory tract infection $(\mathrm{n}=13)$, gastrointestinal tract surgery $(n=11)$, and other causes $(n=7)$. Patients were excluded from the study if they had illness duration greater than 24 hours, evidence of hepatic or renal impairment, history of endocrinopathy, or if they had received steroid treatment. Blood samples for serum cortisol concentrations were collected on admission to the intensive care unit and at regular intervals thereafter for 24 hours. Blood samples were centrifuged, separated, and stored at $-20^{\circ} \mathrm{C}$ prior to assay.

The study was approved by the University College Hospitals Committees on the Ethics of Human Research and the Research Ethics Committees of Great Ormond Street Hospital for Children NHS Trust/Institute of Child Health. Informed written consent was obtained in all cases from parents. 
ASSAYS

Serum cortisol was measured using a solid phase radioimmunoassay (Coat-A-Count, DPC, Los Angeles, California) with a sensitivity of $6 \mathrm{nmol} / 1$, within assay coefficients of variation of $5.7 \%$ and $2.6 \%$ at serum concentrations of $28 \mathrm{nmol} / 1$ and $552 \mathrm{nmol} / 1$ respectively, and between assay coefficients of variation of $6.3 \%$ and $4.5 \%$ at serum concentrations of 138 $\mathrm{nmol} / \mathrm{l}$ and $276 \mathrm{nmol} / 1$ respectively.

\section{STATISTICAL ANALYSIS}

Data obtained from CYP21 deficient patients were natural $\log \left(\log _{\mathrm{e}}\right)$ transformed prior to statistical analysis. The relation between time and the transformed data was described by linear regression. The half life of cortisol was calculated by dividing $0.693\left(\log _{\mathrm{e}} 2\right)$ by the slope of the regression line.

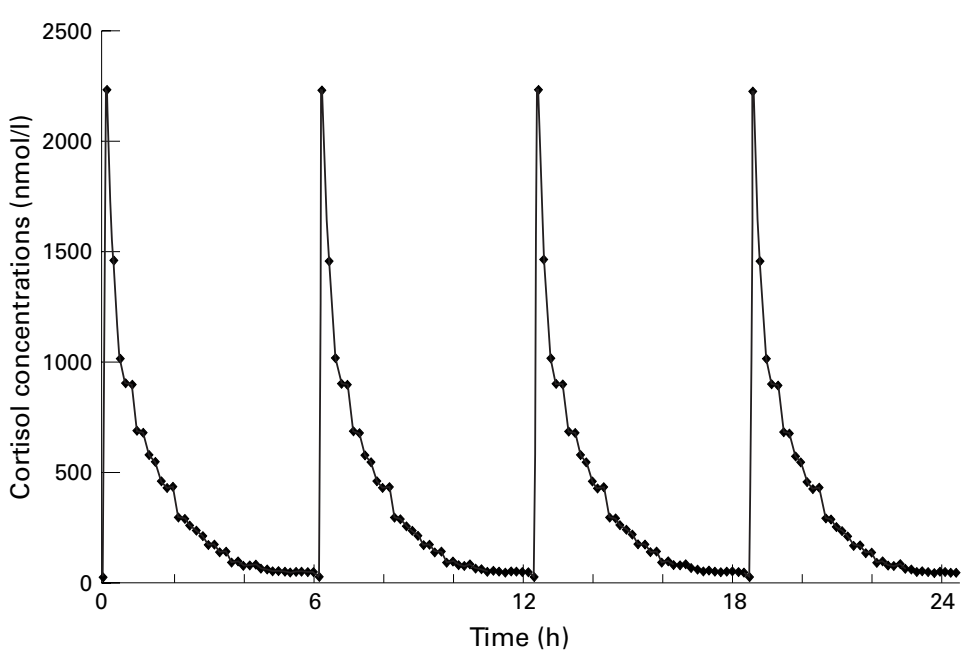

Figure 1 Hypothetical 24 hour cortisol profile in a patient with congenital adrenal hyperplasia caused by 21-hydroxylase deficiency, if intravenous administration of hydrocortisone bolus in a dose of $15 \mathrm{mg} / \mathrm{m}^{2}$ was to be given every six hours.

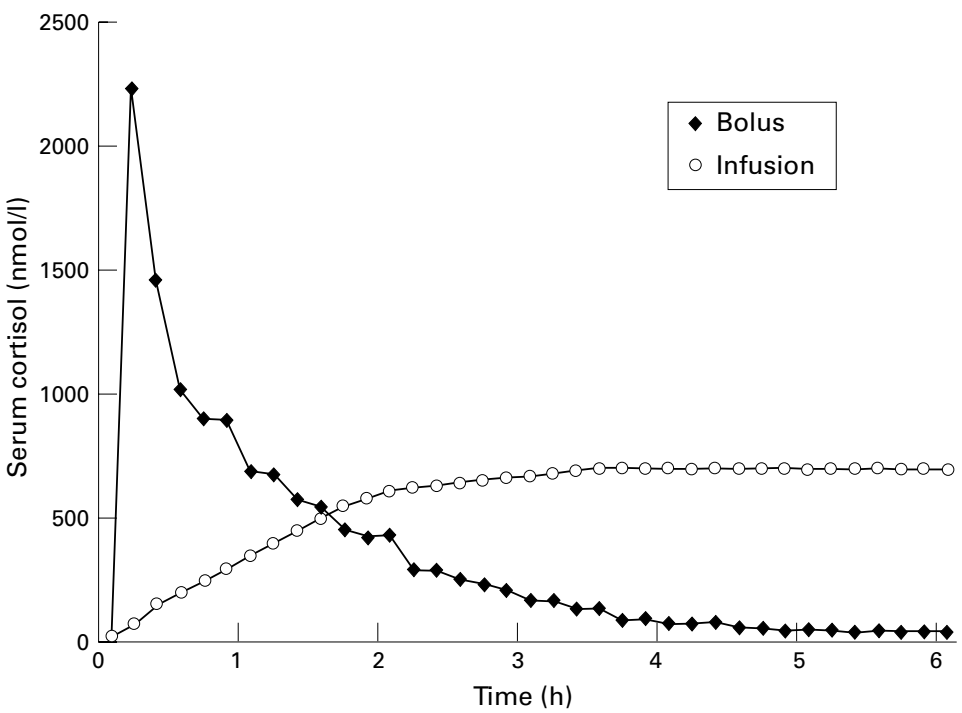

Figure 2 Serum total cortisol concentrations expected to be achieved following constant rate infusion of hydrocortisone, if target cortisol concentration is $727 \mathrm{nmol} / \mathrm{l}$. A single intravenous bolus of hydrocortisone is required to provide adequate glucocorticoid cover during the first 4-6 hours. The desired serum cortisol steady state concentration can be derived from the equation: maintenance dose $(\mathrm{mg} / \mathrm{min})=$ clearance $(\mathrm{ml} / \mathrm{min}) \times$ target concentration $(\mathrm{mg} / \mathrm{ml}){ }^{6}$

\section{Results}

CYP21 DEFICIENT PATIENTS

After administration of the intravenous bolus of hydrocortisone, serum cortisol concentrations rose rapidly and reached a mean peak of $1648.3 \mathrm{nmol} / 1$ (SD 511.9) within 10 minutes. The maximum cortisol concentration $\left(\mathrm{C}_{\max }\right)$ attained was $2700 \mathrm{nmol} / 1$. Cortisol concentrations declined monoexponentially thereafter to reach undetectable concentrations four hours after administration and remained greater than $450 \mathrm{nmol} / 1$ for an average of only 2.5 hours. The half life of cortisol was 83.5 (SD 21.6) minutes.

CRITICALLY ILL PATIENTS

On admission to the paediatric intensive care unit, mean serum cortisol concentration was $727 \mathrm{nmol} / 1$ (SD 426.1) and $\mathrm{C}_{\max } 1700 \mathrm{nmol} / 1$. The majority of critically ill patients $(87 \%)$ had cortisol concentrations exceeding $450 \mathrm{nmol} / 1$. Cortisol concentrations remained raised during the first 24 hours, mean concentration at 24 hours being $515 \mathrm{nmol} / 1$ (SD 264.1).

\section{Discussion}

The above findings show the well documented rise in serum cortisol concentrations in healthy children in response to the stress of critical illness, and highlight the importance of optimal glucocorticoid substitution in critically ill CYP21 deficient patients, who are unable to mount a satisfactory cortisol response to stress. The importance of adequate glucocorticoid substitution in critically ill CYP21 deficient patients is further supported by studies that examined the outcome of children with septic shock in relation to adrenal function. Children with less than $200 \mathrm{nmol} / 1$ increment in serum cortisol concentrations following ACTH stimulation have a higher risk of mortality and require higher doses of inotropes to maintain haemodynamic stability. ${ }^{3}$ Glucocorticoid status is also an important factor in determining outcome in critically ill adults. ${ }^{45}$

Our observations indicate that treatment of critically ill CYP21 deficient patients with intermittent boluses of hydrocortisone (15 $\mathrm{mg} / \mathrm{m}^{2}$ given six hourly) would maintain cortisol concentrations within the range observed in critical illness $(>450 \mathrm{nmol} / \mathrm{l})$ for 10 out of 24 hours and would result in undetectable cortisol concentrations for eight hours (fig 1). Although the hydrocortisone doses used in emergency situations may be higher than the dose used in our study and may result in higher peak cortisol concentrations, the relatively short half life predetermines rapid elimination of cortisol.

It appears, therefore, that to reproduce the response of critical illness in unwell CYP21 deficient patients, either a sustained release formulation of hydrocortisone (not yet available) or a constant rate infusion of hydrocortisone is required. As target steady state concentrations are achieved 3-5 half lives after the initiation of a constant rate infusion, this latter approach should require an intravenous hydrocortisone bolus to provide adequate glucocorticoid cover in the immediate post-stress 
$\operatorname{period}^{6}$ (fig 2). Further studies are required to establish the clinical efficacy of such a management protocol in critically ill CYP21 deficient patients.

We thank our colleagues, Dr J Britto and Dr M Levin, Paediatric Intensive Care Unit, St Mary's Hospital, London, for their permission to recruit patients under their care.

1 New MI. Diagnosis and management of congenital adrenal hyperplasia. Annu Rev Med 1998;49:311-28.
2 Swerdlow AJ, Higgins CD, Brook CGD, et al. Mortality in patients with congenital adrenal hyperplasia: a cohort study. I Pediatr 1998;133:516-20.

3 Hatherill M, Tibby SM, Hillard $\mathrm{T}$, et al. Adrenal insufficiency in septic shock. Arch Dis Child 1999;80:51-5.

4 Bollaert PE, Charpentier C, Levy B, et al. Reversal of late septic shock with supraphysiologic doses of hydrocortisone. Crit Care Med 1998;26:645-50.

5 Briegel J, Forst H, Haller M, et al. Stress doses of hydrocortisone reverse hyperdynamic septic shock: a prospective, randomized, double-blind, single-center study. Crit Care Med 1999;27:723-32.

6 Birkett DJ. Designing dose regimens. In: Pharmacokinetics. Australia: McGraw-Hill, 1998:55-6.

\section{Pneumococcal conjugate vaccine and otitis media}

Up to $50 \%$ of acute otitis media is caused by pneumococci. Conjugate vaccines, you would think, should be able to make inroads into this number and the morbidity and economic costs associated with the disease. A trial in Finland (New England fournal of Medicine 2001;344:403-9), however, has shown no clear cut reduction in the total number of cases.

A total of 1662 infants were randomised to receive either heptavalent pneumococcal polysaccharide conjugate vaccine (conjugated with the non-toxic diphtheria toxin analogue CRM 197) or hepatitis B vaccine (controls) along with routine vaccines at 2, 4, 6, and 12 months of age. They were followed up for between six and 24 months, during which time there were 1251 episodes of acute otitis media in the pneumococcal vaccine group and 1345 in the controls. The rate of acute otitis media was 1.16 episodes/child-year in the pneumococcal vaccine group and 1.24 episodes/child-year in the control group (vaccine efficacy $6 \%(95 \%$ confidence interval $-4 \%$ to $+16 \%)$ ). (A Californian trial of the same vaccine had shown an efficacy of $7 \%$ against acute otitis media.)

There were 414 episodes of pneumococcal acute otitis media (confirmed by culture of middle ear fluid) in the control group and 271 in the pneumococcal vaccine group (34\% reduction). Acute otitis media caused by vaccine serotypes was reduced by $57 \%$ and that caused by cross-reacting serotypes by $51 \%$. Acute otitis media caused by other serotypes of pneumococci was increased by $33 \%$ in the pneumococcal vaccine group. Recurrent acute otitis media was reduced by $9 \%$ overall.

The conjugate vaccine reduced the occurrence of acute otitis media caused by vaccine serotypes and cross-reacting serotypes and possibly reduced the total number of episodes of acute otitis media. They calculated that the vaccine could prevent 1.2 million cases of acute otitis media in the USA each year (but the $95 \%$ confidence limits range from 3.2 million cases prevented to 0.8 million cases caused). Use of the vaccine was followed by an increase in acute otitis media caused by non-vaccine, non-cross-reacting, pneumococcal serotypes.

ARCHIVIST 